\title{
Fixed points of hesitant fuzzy set-valued maps with applications
}

\begin{abstract}
Shagari M.S. ${ }^{1, \otimes}$, Azam A. $^{2}$
In this paper, the notion of hesitant fuzzy fixed points is introduced. To this end, we define Suzuki-type $(\alpha, \beta)$-weak contractions in the framework of hesitant fuzzy set-valued maps, thereby establishing some corresponding fixed point theorems. The presented concept herein is an extension of fuzzy set-valued and multi-valued mappings in the corresponding literature. Examples are provided to support the assertions and generality of our obtained ideas. Moreover, one of our results is applied to investigate sufficient conditions for existence of a class of functional equation arising in dynamic programming.

Key words and phrases: hesitant fuzzy set, hesitant fuzzy set-valued mapping, fixed point, weak contraction.
\end{abstract}

${ }^{1}$ Ahmadu Bello University, Samaru Campus, Community Market, 810211, Zaria, Nigeria

${ }^{2}$ COMSATS University Islamabad, Park Rd, Islamabad, Islamabad Capital Territory 45550, Pakistan

$\triangle$ Corresponding author

E-mail: shagaris@ymail.com (Shagari M.S.), akbarazameyahoo.com (Azam A.)

\section{Introduction and preliminaries}

The classical Banach contraction theorem (see [3]) is one of the extremely useful results in nonlinear functional analysis. In the setting of a metric space, this theorem is stated as follows.

Theorem 1. Let $(X, d)$ be a complete metric space and $T: X \longrightarrow X$ be a contraction, i.e. there exists $\gamma \in(0,1)$ such that

$$
d(T x, T y) \leq \gamma d(x, y), \text { for all } x, y \in X
$$

Then:

(i) $T$ has a unique fixed point $u$ in $X$;

(ii) the Picard iteration $\left\{x_{n}\right\}_{n=0}^{\infty}$, given by $x_{n+1}=T x_{n}, n=0,1,2, \ldots$, converges to $u$ for any $x_{0}$ in $X$.

Theorem 1 has several generalizations, see, for example, $[2,8,11,17,19,22]$ and the references therein. The two well-known drawbacks of Theorem 1 are:

(A) the contractive condition in (1) compels $T$ to be continuous;

(B) the theorem cannot characterize metric completeness of $X$.

Problems (A)-(B) were resolved affirmatively by R. Kannan [16]. Recall that a mapping $T$ (not necessarily continuous) on a metric space $X$ is said to be a Kannan contraction, if there exists an $\gamma \in[0,1 / 2)$ such that

$$
d(T x, T y) \leq \gamma[d(x, T x)+d(y, T y)] \quad \text { for all } \quad x, y \in X .
$$


This achievement in Kannan contraction was first noted by P.V. Subrahmanyam [22] that a metric space $X$ is complete if and only if every Kannan contraction on $X$ has a fixed point. Following (2), more than a handful of papers were devoted to studying fixed point theorems for classes of contractive type conditions that do not require the continuity of $T$; see, for instance [9]. However, researchers noticed that Kannan's theorem is not a generalization of Theorem 1. Along the way, the notion of weak contraction was introduced by V. Berinde [5]. The idea generalized the well-celebrated fixed point theorems due to S. Banach [3], N. Chandra, M.C. Joshi, and N.K. Singh [9] and many others. For our purpose, we recall from [5, Definition 1] for convenience as follows.

Definition 1. Let $(X, d)$ be a metric space. A mapping $T: X \longrightarrow X$ is called weak contraction if there exists a constants $\gamma \in(0,1)$ and $K \geq 0$ such that $d(T x, T y) \leq \gamma d(x, y)+K d(y, T x)$ for all $x, y \in X$.

Thereafter, in 2007, M. Berinde and V. Berinde [6] extended the concept of weak contraction from the case of single-valued mappings to multi-valued mappings and established some convergence theorems for the Picard iteration in connection with multi-valued weak contraction.

Definition 2. Let $(X, d)$ be a metric space and $T: X \longrightarrow C B(X)$ be a multi-valued mapping. Then $T$ is called a multi-valued weak contraction or multi-valued $(\theta, K)$-weak contraction if and only if there exist constants $\theta \in(0,1)$ and $K \geq 0$ such that $H(T x, T y) \leq$ $\theta d(x, y)+K d(y, T x)$ for all $x, y \in X$.

For some recent results regarding weak-contractions and related fixed point theorems, we refer P. Borisut et al. [7] and the references therein. Furthermore, a notable attempt at resolving problems (A)-(B) was presented in 2008 by T. Suzuki [23]. The following is known in the literature as Suzuki fixed point theorem.

Theorem 2 ([23]). Let $(X, d)$ be a complete metric space and $T$ be a mapping on $X$. Define a nondecreasing function $r$ from $[0,1)$ onto $(1 / 2,1]$ by

$$
r(t)= \begin{cases}1, & \text { if } \quad 0 \leq t \leq(\sqrt{5}-1) / 2, \\ (1-t) / r^{2}, & \text { if } \quad(\sqrt{5}-1) / 2 \leq r \leq 1 / \sqrt{2}, \\ 1 /(1+t), & \text { if } 1 / \sqrt{2} \leq r<1 .\end{cases}
$$

Assume that there exists $t \in[0,1)$ such that $r(t) d(x, T x) \leq d(x, y)$ implies $d(T x, T y) \leq r d(x, y)$ for all $x, y \in X$. Then $T$ has a unique fixed point $u$ in $X$. Moreover, $T^{n} x \rightarrow u$ as $n \rightarrow \infty$ for all $x \in X$.

An interesting improvement of Suzuki fixed point theorem in the setting of multivalued mappings is due to D. Đorić and R. Lazović [12]. In subsequent section, we shall derive the main result in [12] and others as consequences of our result.

On the other hand, one of the challenges in mathematical modeling of practical phenomena concerns the indeterminacy induced by our inability to categorize events with adequate precision. It has been noted that classical mathematics cannot cope successfully with imprecisions. As an attempt at reducing the forgoing obstacles, the notion of fuzzy sets was initiated by L.A. Zadeh [26] in 1965 as one of the uncertainty approaches and tools to construct mathematical models compatible with real world problems in engineering, life science, economics, 
medicine, language theory, to mention but a few. Meanwhile, the basic ideas of fuzzy sets have been generalized in different directions. In 1981, S. Heilpern [15] used the notion of fuzzy set to initiate a class of fuzzy mappings which generalizes the concept of set-valued mappings. In the process, he provided the existence of fuzzy fixed point theorem which is an improvement of Banach contraction theorem [3] and Nadler's fixed point theorem [19] for multi-valued mappings. M. Frigon and D. O'Regan [13] improved Heilpern fixed point theorem under a contractive condition for 1-level sets, where the 1-level sets are not assumed to be convex and compact. Along the line, H.M. Abu-Donia [1] proved some common fixed point theorems for fuzzy mappings defined on a complete metric space under $\varphi$-contraction. His investigation is related to fuzzy forms of Hausdorff metric which is an important tool for computing Hausdorff dimensions. Thereafter, several researchers studied the existence of fixed points and common fixed points of fuzzy mappings satisfying various types of contractive conditions on metric and quasi metric spaces (see, e.g. $[14,17,18]$ and the references therein).

In 2010, V. Torra [24] introduced an extension of fuzzy sets, called hesitant fuzzy sets (HFSs) motivated by the common complexity that usually appears when the degree of membership of an element must be obtained. HFSs have allured the attentions of several researchers in a short period of time due to the fact that hesitant scenarios are common in realworld problems. For expository note on some vital developments in the theory of HFSs, the interested reader may consult R.M. Rodriguez [21].

The main aim of this paper is to extend the idea of multi-valued weak contraction from the case of crisp mappings to hesitant fuzzy set-valued maps. To this end, motivated by the results of T. Suzuki [23], D. Đorić and R. Lazović [12], we define the notion of Suzuki-type $(\alpha, \beta)$-weak contractions in the setting of hesitant fuzzy set-valued maps and investigate sufficient conditions for existence of hesitant fuzzy fixed point for such contractions. In the framework of hesitant fuzzy set-valued maps, the obtained result is a generalization of the related concepts of fuzzy sets and multivalued mappings. In addition, an application in dynamic programming is considered to highlight a possible utility of our ideas herein.

\subsection{Fuzzy and multivalued mappings}

Let $(X, d)$ be a metric space. We denote by $C B(X)$ the class of all nonempty, closed and bounded subsets of $X$. Let $H(.,$.$) be the Hausdorff metric on C B(X)$ induced by the metric $d$,

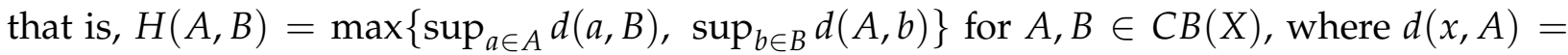
$\inf \{d(x, a): a \in A\}$. A point $u$ in $X$ is a fixed point of a multi-valued mapping $T: X \rightarrow C B(X)$ if $u \in T u$.

Recall that an ordinary subset $A$ of $X$ is determined by its characteristic function $\chi_{A}: A \rightarrow\{0,1\}$, defined by

$$
\chi_{A}(x)=\left\{\begin{array}{lll}
1, & \text { if } & x \in A, \\
0, & \text { if } & x \notin A .
\end{array}\right.
$$

The value $\chi_{A}(x)$ specifies wether an element belongs to $A$ or not. This idea is used to define fuzzy sets by allowing an element $x \in A$ to assume any possible value in the interval $[0,1]$. Thus, a fuzzy set $A$ in $X$ is a set of ordered pairs given as $A=\left\{\left(x, \mu_{A}(x)\right): x \in X\right\}$, where $\mu_{A}: X \rightarrow[0,1]=I$ and $\mu_{A}(x)$ is called the membership function of $x$ or the degree to which $x \in X$ belongs to the fuzzy set $A$. The $\alpha$-level set of a fuzzy set $A$ in $X$ is denoted by 
$[A]_{\alpha}$, and is defined as follows

$$
[A]_{\alpha}= \begin{cases}\overline{\{x \in X: A(x)>0\}}, & \text { if } \quad \alpha=0, \\ \{x \in X: A(x) \geq \alpha\}, & \text { if } \quad \alpha \in(0,1],\end{cases}
$$

where by $\bar{M}$, we mean the closure of the crisp set $M$. We denote the family of fuzzy sets in $X$ by $I^{X}$.

Throughout this paper, we take $(0,1]=I^{+1}$ and $(0,1)=I_{-1}$. Let $X$ be an arbitrary set and $Y$ be a metric space. A mapping $T: X \rightarrow I^{Y}$ is called fuzzy mapping. A fuzzy mapping $T$ is a fuzzy subset of $X \times Y$. The function value $T(x)(y)$ is the degree of membership of $y$ in $T(x)$. An element $u$ in $X$ is said to be a fuzzy fixed point of $T$ if there exists an $\alpha \in I^{+1}$ such that $u \in[T u]_{\alpha}$.

We denote the set of all fixed points of $T$ by $\mathcal{F}_{i x}(T)$.

\subsection{Hesitant fuzzy sets}

V. Torra [24] introduced an extension of fuzzy sets, called hesitant fuzzy set (HFS) to handle hesitant situations, which were not efficiently managed by the earlier tools such as fuzzy and intuitionistic fuzzy sets. Precisely, HFS deals with those situations in which several values are possible for the definition of a membership function. Hence, HFS is defined in terms of a function that returns a set of membership values for each element in the domain.

Definition 3 ([24]). Let $X$ be a universal set. A HFS $A$ on $X$ is a function that when applied to $X$ returns a finite subset of $[0,1]$.

Afterward, M. Xia and Z. Xu [25] complemented Definition 3 by providing the mathematical representation of a HFS as follows: $A=\left\{\left(x, h^{A}(x)\right): x \in X\right\}$, where $h^{A}(x)$ is a finite subset of $[0,1]$, and denotes the possible membership degrees of $x \in X$ to the set $A$. For convenience, M. Xia and Z. Xu [25] noted $h=h_{A}(x)$ and named it as hesitant fuzzy element (HFE) of $A$. In other words, a HFS is a set of subset of $[0,1]$, one for each element of the reference set $X$. On the other hand, a HFE is one of such sets, corresponding to each $x \in X$.

A HFS can also be constructed from a set of fuzzy sets.

Definition 4 ([24]). Let $M=\left\{\mu_{1}, \mu_{2}, \ldots, \mu_{n}\right\}$ be a set of $n$ membership functions. The HFS associated with $M, h_{M}$, is defined by $h_{M}(x)=\bigcup_{\mu \in M}\{\mu(x)\}$.

To establish an order between HFSs, M. Xia and Z. Xu [25] introduced a comparison law by defining a score function, which is defined under the following assumptions:

(i) the values of all the HFEs are arranged in an increasing order;

(ii) the HFEs have the same length when they are compared. Thus, if any two HFEs have different length, the shorter one will be extended by adding the maximum element until both HFEs have the same length.

Definition 5 ([25]). Let $h$ be an HFE, the score function of $h$ is defined by $s(h)=(1 / l(h)) \sum_{\lambda \in h} \lambda$, where $l(h)$ is the number of element(s) in $h$ and $s(h) \in[0,1]$.

T. Rashid and I. Beg [20] introduced the concept of $\alpha$-cut of a HFS as follows.

Definition 6 ([20]). Given $\alpha \in I^{+1}$, the crisp subset of $X$ defined by $h_{\alpha}^{A}=\left\{t \in X: s\left(h^{A}(t)\right) \geq \alpha\right\}$, is said to be the $\alpha$-cut of the HFS A on X. 
Recall that HFS is a generalization of intuitionistic fuzzy set. Following the idea of $(\alpha, \beta)$-cut of intuitionistic fuzzy set (see [27]) and motivated by Definition 6, we give the following notion.

Definition 7. Given $(\alpha, \beta) \in(0,1] \times[0,1)$, the crisp subset of $X$ defined as

$$
h_{(\alpha, \beta)}^{A}=\left\{t \in X: s\left(h^{A}(t)\right) \geq \alpha\right\} \bigcup\left\{t \in X: s\left(h^{A}(t)\right) \leq \beta\right\},
$$

is called the $(\alpha, \beta)$-level set of the HFS $A$ on $X$.

Clearly, Definition 7 reduces to Definition 6 if $s\left(h^{A}(t)\right)>\beta$ for all $t \in X$.

Example 1. Let $X=[0,1]$ and $h: X \rightarrow[0,1]$ be defined by

$$
h(t)= \begin{cases}\{0,0.6,0.7,0.8\}, & \text { if } 0 \leq t \leq 1 / 10 \\ \{0.2,0.3,0.4,0.5\}, & \text { if } 1 / 10<t \leq 1 / 5, \\ \{0.2,0.6,0.8,1\}, & \text { if } 1 / 5<t \leq 1\end{cases}
$$

For $0 \leq t \leq 1 / 10, s(h)=(0.8+0.7+0.6+0) / 4=0.53$; for $1 / 10<t \leq 1 / 5, s(h)=$ $(0.5+0.4+0.3+0.2) / 4=0.35 ;$ and for $1 / 5<t \leq 1, s(h)=(1+0.8+0.6+0.2) / 4=0.65$.

Let $\alpha=0.62$ and $\beta=0.4$, then $h_{(\alpha, \beta)}^{A}=(1 / 10,1 / 5] \cup(1 / 5,1]=(1 / 10,1]$.

Example 2. Let $X=\{1,2,3,4,5,6\}$ and $h: X \rightarrow[0,1]$ be defined by

$$
h(t)= \begin{cases}\{0.2,0.3,0.4\}, & \text { if } t \in\{1,2,3\}, \\ \{0.5,0.6,0.9\}, & \text { if } t \in\{4,5\}, \\ \{0.1,0.7,0.8\}, & \text { if } t=6 .\end{cases}
$$

For $t \in\{1,2,3\}, s(h)=0.3$; for $t \in\{4,5\}, s(h)=0.67$; and for $t=6, s(h)=0.53$.

Let $\alpha=0.6$ and $\beta=0.1$, then $h_{(\alpha, \beta)}^{A}=\{4,5\} \cup \varnothing=\{4,5\}=h_{\alpha}^{A}$.

Definition 8. Let $X$ be a universal set and $F_{H}(X)$ denotes the set of all hesitant fuzzy sets on $X$. A set-valued map $T: X \rightarrow F_{H}(X)$ is called hesitant fuzzy mapping on $X$.

Note that if $T$ is a hesitant fuzzy mapping on $X$, then $T x: X \rightarrow[0,1]$ is an HFE, for each $x \in X$ and $(T x)(t)$ is a finite subset of $[0,1]$ for each $t \in X$.

Definition 9. Let $T$ be a hesitant fuzzy set on $X$. Given $(\alpha, \beta) \in(0,1] \times[0,1)$, the crisp subset of $X$ defined as $(T x)_{(\alpha, \beta)}=\{t \in X: s((T x)(t)) \geq \alpha\} \cup\{t \in X: s((T x)(t)) \leq \beta\}$, is said to be the $(\alpha, \beta)$-level set of $T$, where $s((T x)(t))$ is the score function of $T$. A point $u \in X$ is called hesitant fuzzy fixed point of $T$ if there exists $(\alpha, \beta) \in(0,1] \times[0,1)$ such that $u \in(T u)_{(\alpha, \beta)}$.

Throughout the paper, the function $r: I_{-1} \rightarrow(1 / 2,1]$ is defined as

$$
r(\alpha)= \begin{cases}1, & \text { if } \quad 0<\alpha<1 / 2 \\ 1-\alpha, & \text { if } 1 / 2 \leq \alpha<1\end{cases}
$$

For $x, y \in X$ and $(\alpha, \beta) \in(0,1] \times[0,1)$, define

$$
\begin{aligned}
& \vee(x, y)=\max \left\{d(x, y), d\left(x,(T x)_{(\alpha, \beta)}\right), d\left(y,(T y)_{(\alpha, \beta)}\right),\right. \\
& \left.\frac{d\left(x,(T y)_{(\alpha, \beta)}\right)+d\left(y,(T x)_{(\alpha, \beta)}\right)}{2}, \frac{d\left(x,(T x)_{(\alpha, \beta)}\right) d\left(y,(T y)_{(\alpha, \beta)}\right)}{1+d(x, y)}\right\}, \\
& \wedge(x, y)=\min \left\{d\left(x,(T x)_{(\alpha, \beta)}\right), d\left(y,(T x)_{(\alpha, \beta)}\right), \frac{d\left(x,(T x)_{(\alpha, \beta)}\right) d\left(y,(T y)_{(\alpha, \beta)}\right)}{1+d(x, y)}\right\} .
\end{aligned}
$$




\section{Hesitant fuzzy fixed points of Suzuki-type $(\alpha, \beta)$-weak contractions}

In this section, we present hesitant fuzzy fixed theorem of Suzuki-type $(\alpha, \beta)$-weak contractions and then obtain some associated consequences. Motivated by Definitions 1 and 2 as well as Theorem 2, we introduce the following concept.

Definition 10. A hesitant fuzzy mapping $T: X \rightarrow F_{H}(X)$ is called Suzuki-type $(\alpha, \beta)$-weak contraction if for all $x, y \in X$ with $x \neq y$, there exists some $(\alpha, \beta) \in(0,1] \times[0,1)$ such that $r(\alpha) d\left(x,(T x)_{(\alpha, \beta)}\right) \leq d(x, y)$ implies $H\left((T x)_{(\alpha, \beta)},(T y)_{(\alpha, \beta)}\right) \leq \alpha \vee(x, y)+\beta \wedge(x, y)$.

Theorem 3. Let $(X, d)$ be a complete metric space and $T: X \rightarrow F_{H}(X)$ be a Suzuki-type $(\alpha, \beta)$-weak contraction. Assume that for each $x \in X$, there exists $(\alpha, \beta) \in(0,1] \times[0,1)$ such that $(T x)_{(\alpha, \beta)}$ is a nonempty closed and bounded subset of $X$. Then $\mathcal{F}_{i x}(T) \neq \varnothing$.

Proof. Let $(\alpha, \beta) \in(0,1] \times[0,1)$ be such that $0<\alpha \leq \alpha_{1}<1, x_{1} \in X$ and $\rho=1 / \sqrt{\alpha}$. Then by hypothesis, $\left(T x_{1}\right)_{(\alpha, \beta)}$ is nonempty. Therefore, we can find $x_{2} \in X$ such that $x_{2} \in\left(T x_{1}\right)_{(\alpha, \beta)}$. Since $\rho>1$, choose $x_{3} \in\left(T x_{2}\right)_{(\alpha, \beta)}$ such that $d\left(x_{2}, x_{3}\right) \leq \rho H\left(\left(T x_{1}\right)_{(\alpha, \beta)},\left(T x_{2}\right)_{(\alpha, \beta)}\right)$. If $x_{1}=x_{2}$, then $x_{1} \in\left(T x_{1}\right)_{(\alpha, \beta)}$ for some $(\alpha, \beta) \in(0,1] \times[0,1)$, and the theorem is proved. Assume that $x_{1} \neq x_{2}$. Since $r(\alpha) \leq 1$, therefore, by hypothesis, $r(\alpha) d\left(x_{1},\left(T x_{1}\right)_{(\alpha, \beta)}\right) \leq d\left(x_{1},\left(T x_{1}\right)_{(\alpha, \beta)}\right) \leq$ $d\left(x_{1}, x_{2}\right)$ implies

$$
\begin{aligned}
d\left(x_{2}, x_{3}\right) \leq & \rho H\left(\left(T x_{1}\right)_{(\alpha, \beta)},\left(T x_{2}\right)_{(\alpha, \beta)}\right) \leq \frac{1}{\sqrt{\alpha}}(\alpha \vee(x, y)+\beta \wedge(x, y)) \\
\leq & \sqrt{\alpha} \max \left\{d\left(x_{1}, x_{2}\right), d\left(x_{1},\left(T x_{1}\right)_{(\alpha, \beta)}\right), d\left(x_{2},\left(T x_{2}\right)_{(\alpha, \beta)}\right),\right. \\
& \left.\frac{d\left(x_{1},\left(T x_{2}\right)_{(\alpha, \beta)}\right)+d\left(x_{2},\left(T x_{1}\right)_{(\alpha, \beta)}\right)}{2}, \frac{d\left(x_{1},\left(T x_{1}\right)_{(\alpha, \beta)}\right) d\left(x_{2},\left(T x_{2}\right)_{(\alpha, \beta)}\right)}{1+d\left(x_{1}, x_{1}\right)}\right\} \\
+ & \frac{\beta}{\sqrt{\alpha}} \min \left\{d\left(x_{1},\left(T x_{1}\right)_{(\alpha, \beta)}\right), d\left(x_{2},\left(T x_{1}\right)_{(\alpha, \beta)}\right), \frac{d\left(x_{1},\left(T x_{1}\right)_{(\alpha, \beta)}\right) d\left(x_{2},\left(T x_{2}\right)(\alpha, \beta)\right.}{1+d\left(x_{1}, x_{2}\right)}\right\} \\
\leq & \sqrt{\alpha} \max \left\{d\left(x_{1}, x_{2}\right), d\left(x_{2}, x_{3}\right), \frac{d\left(x_{1}, x_{3}\right)+d\left(x_{2}, x_{2}\right)}{2}, \frac{d\left(x_{1}, x_{2}\right) d\left(x_{2}, x_{3}\right)}{1+d\left(x_{1}, x_{2}\right)}\right\} \\
& +\frac{\beta}{\sqrt{\alpha}} \min \left\{d\left(x_{1}, x_{2}\right), d\left(x_{2}, x_{2}\right), \frac{d\left(x_{1}, x_{2}\right) d\left(x_{2}, x_{3}\right)}{1+d\left(x_{1}, x_{2}\right)}\right\} \\
\leq & \sqrt{\alpha} \max \left\{d\left(x_{1}, x_{2}\right), d\left(x_{2}, x_{3}\right), \frac{d\left(x_{1}, x_{2}\right)+d\left(x_{2}, x_{3}\right)}{2}\right\} \leq \sqrt{\alpha} \max \left\{d\left(x_{1}, x_{2}\right), d\left(x_{2}, x_{3}\right)\right\} .
\end{aligned}
$$

If $d\left(x_{1}, x_{2}\right) \leq d\left(x_{2}, x_{3}\right)$, then from (3), we have $d\left(x_{2}, x_{3}\right) \leq \sqrt{\alpha} d\left(x_{2}, x_{3}\right)<d\left(x_{2}, x_{3}\right)$, a contradiction. Hence, $d\left(x_{1}, x_{2}\right)>d\left(x_{2}, x_{3}\right)$ and (3) becomes $d\left(x_{2}, x_{3}\right) \leq \sqrt{\alpha} d\left(x_{1}, x_{2}\right) \leq \sqrt{\alpha_{1}} d\left(x_{1}, x_{2}\right)$. Continuing in this fashion, we generate a sequence $\left\{x_{n}\right\}_{n \in \mathbb{N}}$ in $X$ such that $x_{n+1} \in\left(T x_{n}\right)_{(\alpha, \beta)}$ and $d\left(x_{n}, x_{n+1}\right) \leq \sqrt{\alpha_{1}} d\left(x_{n-1}, x_{n}\right)$, from which we have

$$
\sum_{n=1}^{\infty} d\left(x_{n}, x_{n+1}\right) \leq \sum_{n=1}^{\infty}\left(\sqrt{\alpha_{1}}\right)^{n-1} d\left(x_{1}, x_{2}\right)<\infty
$$

By a standard argument, we conclude that $\left\{x_{n}\right\}_{n \in \mathbb{N}}$ is a Cauchy sequence in $X$. The completeness of $X$ implies that there exists $u \in X$ such that $x_{n} \rightarrow u$ as $n \rightarrow \infty$.

Claim: for all $u \neq z$, we have

$$
d\left(u,(T z)_{(\alpha, \beta)} \leq \alpha \max \left\{d(u, z), d\left(z,(T z)_{(\alpha, \beta)}\right\} .\right.\right.
$$


Since $x_{n} \rightarrow u$ as $n \rightarrow \infty$, there exists a positive integer $m$ such that $5 d\left(x_{n}, u\right) \leq d(u, z)$ for all $n \geq m$. Given that $x_{n+1} \in\left(T x_{n}\right)_{(\alpha, \beta)}$, we get

$r(\alpha) d\left(x_{n},\left(T x_{n}\right)_{(\alpha, \beta)}\right) \leq d\left(x_{n},\left(T x_{n}\right)_{(\alpha, \beta)}\right) \leq d\left(x_{n}, x_{n+1}\right) \leq d\left(x_{n}, u\right)+d\left(u, x_{n+1}\right) \leq(2 / 5) d(u, z)$.

Thus, for $n \geq m$, we have

$$
r(\alpha) d\left(x_{n},\left(T x_{n}\right)_{(\alpha, \beta)}\right) \leq \frac{2}{5} d(u, z) \leq d(u, z)-\frac{1}{5} d(u, z) \leq d(u, z)-d\left(u, x_{n}\right) \leq d\left(x_{n}, z\right) .
$$

Hence, (5) implies

$$
\begin{aligned}
& d\left(x_{n+1},(T z)_{(\alpha, \beta)}\right) \leq H\left(\left(T x_{n}\right)_{(\alpha, \beta)},(T z)_{(\alpha, \beta)}\right) \\
& \leq \alpha \max \{ d\left(x_{n}, z\right), d\left(x_{n},\left(T x_{n}\right)_{(\alpha, \beta)}\right), d\left(z,(T z)_{(\alpha, \beta)}\right), \\
&\left.\frac{d\left(x_{n},(T z)_{(\alpha, \beta)}\right)+d\left(z,\left(T x_{n}\right)_{(\alpha, \beta)}\right)}{2}, \frac{d\left(x_{n},\left(T x_{n}\right)_{(\alpha, \beta)}\right) d\left(z,(T z)_{(\alpha, \beta)}\right)}{1+d\left(x_{n}, z\right)}\right\} \\
&+\beta \min \left\{d\left(x_{n},\left(T x_{n}\right)_{(\alpha, \beta)}\right), d\left(z,\left(T x_{n}\right)_{(\alpha, \beta)}\right), \frac{d\left(x_{n},\left(T x_{n}\right)_{(\alpha, \beta)}\right) d\left(z,(T z)_{(\alpha, \beta)}\right)}{1+d\left(x_{n}, z\right)}\right\} .
\end{aligned}
$$

From (6), we have

$$
\begin{aligned}
d\left(x_{n+1},(T z)_{(\alpha, \beta)}\right) \leq \alpha \max \{ & d\left(x_{n}, z\right), d\left(x_{n}, x_{n+1}\right), d\left(z,(T z)_{(\alpha, \beta)}\right), \\
& \left.\frac{d\left(x_{n}(T z)_{(\alpha, \beta)}\right)+d\left(z, x_{n+1}\right)}{2}, \frac{d\left(x_{n},\left(T x_{n}\right)_{(\alpha, \beta)}\right) d\left(z,(T z)_{(\alpha, \beta)}\right)}{1+d\left(x_{n}, z\right)}\right\} \\
+ & \beta \min \left\{d\left(x_{n}, x_{n+1}\right), d\left(z, x_{n+1}\right), \frac{d\left(x_{n}, x_{n+1}\right) d\left(z,(T z)_{(\alpha, \beta)}\right)}{1+d\left(x_{n}, z\right)}\right\} .
\end{aligned}
$$

As $n \rightarrow \infty$ in (7), we obtain

$$
\begin{aligned}
d\left(u,(T z)_{(\alpha, \beta)}\right) & \leq \alpha \max \left\{d(u, z), d\left(z,(T z)_{(\alpha, \beta)}\right), \frac{d\left(u,(T z)_{(\alpha, \beta)}\right)+d(u, z)}{2}\right\}+\beta \min \{0, d(u, z)\} \\
& \leq \alpha \max \left\{d(u, z), d\left(z,(T z)_{(\alpha, \beta)}\right)\right\} .
\end{aligned}
$$

Now, to show that $u \in(T u)_{(\alpha, \beta)}$, we consider the following two possibilities.

Case (i). $0<\alpha<1 / 2$.

Suppose that for all $(\alpha, \beta) \in[0,1) \times[0,1)$ with $\alpha<1, u \neq p, u \notin(T u)_{(\alpha, \beta)}$ and $p \in(T u)_{(\alpha, \beta)}$ such that $d(p, u)<d\left(u,(T u)_{(\alpha, \beta)}\right)$. Setting $z=p$ in (4), we have

$$
d\left(u,(T p)_{(\alpha, \beta)}\right) \leq \alpha \max \left\{d(u, p), d\left(p,(T p)_{(\alpha, \beta)}\right)\right\}
$$

Now, $r(\alpha) d\left(u,(T u)_{(\alpha, \beta)}\right) \leq d\left(u,(T u)_{(\alpha, \beta)}\right) \leq d(u, p)$ implies 


$$
\begin{aligned}
d\left(p,(T p)_{(\alpha, \beta)}\right) \leq & H\left((T u)_{(\alpha, \beta)},(T p)_{(\alpha, \beta)}\right) \leq \alpha \max \left\{d(u, p), d\left(u,(T u)_{(\alpha, \beta)}\right), d\left(p,(T p)_{(\alpha, \beta)}\right),\right. \\
& \left.\frac{d\left(u,(T p)_{(\alpha, \beta)}\right)+d\left(p,(T u)_{(\alpha, \beta)}\right)}{2}, \frac{d\left(u,(T u)_{(\alpha, \beta)}\right) d\left(p,(T p)_{(\alpha, \beta)}\right)}{1+d(u, p)}\right\} \\
& +\beta \min \left\{d\left(u,(T u)_{(\alpha, \beta)}\right), d\left(p,(T u)_{(\alpha, \beta)}\right), \frac{d\left(u,(T u)_{(\alpha, \beta)}\right) d\left(p,(T p)_{(\alpha, \beta)}\right)}{1+d(u, p)}\right\} \\
\leq & \alpha \max \left\{d(u, p), d\left(p,(T p)_{(\alpha, \beta)}\right), \frac{d\left(u,(T p)_{(\alpha, \beta)}\right)}{2}, \frac{d(u, p) d\left(p,(T p)_{(\alpha, \beta)}\right)}{1+d(u, p)}\right\} \\
& +\beta \min \left\{d(u, p), 0, \frac{d(u, p) d\left(p,(T p)_{(\alpha, \beta)}\right)}{1+d(u, p)}\right\} \\
\leq & \max \left\{d(u, p), d\left(p,(T p)_{(\alpha, \beta)}\right), \frac{d(u, p)+d\left(p,(T p)_{(\alpha, \beta)}\right)}{2}\right\} \\
\leq & \alpha \max \left\{d(u, p), d\left(p,(T p)_{(\alpha, \beta)}\right)\right\} .
\end{aligned}
$$

Assume that $d(u, p) \leq d\left(p,(T p)_{(\alpha, \beta)}\right)$, then from above inequalities, we have $d\left(p,(T p)_{(\alpha, \beta)}\right) \leq$ $\alpha d\left(p,(T p)_{(\alpha, \beta)}\right)<d\left(p,(T p)_{(\alpha, \beta)}\right)$, a contradiction. Hence, $d(u, p)>d\left(p,(T p)_{(\alpha, \beta)}\right)$, and $d\left(p,(T p)_{(\alpha, \beta)}\right) \leq \alpha d(u, p)<d(u, p)$. Therefore, (8) becomes $d\left(u,(T p)_{(\alpha, \beta)}\right) \leq \alpha d(u, p)$. Consequently,

$$
\begin{aligned}
d\left(u,(T u)_{(\alpha, \beta)}\right) & \leq d\left(u,(T p)_{(\alpha, \beta)}\right)+H\left((T p)_{(\alpha, \beta)},(T u)_{(\alpha, \beta)}\right) \\
& \leq d\left(u,(T p)_{(\alpha, \beta)}\right)+\alpha \max \left\{d(u, p), d\left(p,(T p)_{(\alpha, \beta)}\right)\right\} \\
& \leq \alpha d(u, p)+\alpha d(u, p)=2 \alpha d(u, p)<d(u, p)<d\left(u,(T u)_{(\alpha, \beta)}\right)
\end{aligned}
$$

(in view of assumption) yields a contradiction. Therefore, $u \in(T u)_{(\alpha, \beta)}$.

Case (ii). $1 / 2 \leq \alpha<1$.

For this, we shall prove that

$$
\begin{aligned}
H\left((T z)_{(\alpha, \beta)},(T u)_{(\alpha, \beta)}\right) \leq & \alpha \max \left\{d(u, z), d\left(z,(T z)_{(\alpha, \beta)}\right), d\left(u,(T u)_{(\alpha, \beta)}\right),\right. \\
& \left.\frac{d\left(z,(T u)_{(\alpha, \beta)}\right)+d\left(u,(T z)_{(\alpha, \beta)}\right)}{2}, \frac{d\left(z,(T z)_{(\alpha, \beta)}\right) d\left(u,(T u)_{(\alpha, \beta)}\right)}{1+d(u, z)}\right\} \\
+ & \beta \min \left\{d\left(z,(T z)_{(\alpha, \beta)}\right), d\left(u,(T z)_{(\alpha, \beta)}\right), \frac{d\left(u,(T u)_{(\alpha, \beta)}\right) d\left(z,(T z)_{(\alpha, \beta)}\right)}{1+d(u, z)}\right\},
\end{aligned}
$$

holds for all $z \in X$ with $z \neq u$. Now, for all $m \in \mathbb{N}$, there exists $v_{m} \in(T z)_{(\alpha, \beta)}$ such that

$$
d\left(u, v_{m}\right) \leq d\left(u,(T z)_{(\alpha, \beta)}\right)+\frac{1}{5 m} d(u, z) .
$$

Thus, we have

$$
d\left(z,(T z)_{(\alpha, \beta)}\right) \leq d\left(z, v_{m}\right) \leq d(z, u)+d\left(u, v_{m}\right) \leq d(z, u)+d\left(u,(T z)_{(\alpha, \beta)}\right)+\frac{1}{5 m} d(z, u) .
$$

Using (4), we have

$$
d\left(z,(T z)_{(\alpha, \beta)}\right) \leq d(u, z)+\alpha \max \left\{d(u, z), d\left(z,(T z)_{(\alpha, \beta)}\right)\right\}+\frac{1}{5 m} .
$$


If $d(z, u)>d\left(z,(T z)_{(\alpha, \beta)}\right)$, then from $(10)$, we get

$$
d\left(z,(T z)_{(\alpha, \beta)}\right) \leq d(u, z)+\alpha d(u, z)+\frac{1}{5 m} d(u, z)=\left[(1+\alpha)+\frac{1}{5 m}\right] d(u, z),
$$

from which we have

$$
\left[\frac{1}{1+\alpha}\right] d\left(z,(T z)_{(\alpha, \beta)}\right) \leq\left[1+\frac{1}{5(1+\alpha) m}\right] d(u, z) .
$$

Using $r(\alpha)=1-\alpha$, we get

$r(\alpha) d\left(z,(T z)_{(\alpha, \beta)}\right)=(1-\alpha) d\left(z,(T z)_{(\alpha, \beta)}\right) \leq\left(\frac{1}{1+\alpha}\right) d\left(z,(T z)_{(\alpha, \beta)}\right) \leq\left[1+\frac{1}{5(1+\alpha) m}\right] d(u, z)$.

As $m \rightarrow \infty$ in the above inequalities, we have $r(\alpha) d\left(z,(T z)_{(\alpha, \beta)}\right) \leq d(u, z)$. On the other hand, if $d(u, z)<d\left(z,(T z)_{(\alpha, \beta)}\right)$, then (10) gives

$$
d\left(z,(T z)_{(\alpha, \beta)}\right) \leq d(u, z)+\alpha d\left(z,(T z)_{(\alpha, \beta)}\right)+\frac{1}{5 m} d(u, z),
$$

which yields

$$
(1-\alpha) d\left(z,(T z)_{(\alpha, \beta)}\right) \leq\left(1+\frac{1}{5 m}\right) d(u, z) .
$$

As $m \rightarrow \infty$ in the above inequality, we have $(1-\alpha) d\left(z,(T z)_{(\alpha, \beta)}\right) \leq d(u, z)$. This shows that

$$
r(\alpha) d\left(z,(T z)_{(\alpha, \beta)}\right) \leq d(u, z),
$$

which, by Definition 10, implies (9). Moreover, since $x_{n+1} \neq x_{n}$ for all $n \in \mathbb{N}$, then $u \neq x_{n+1}$. Therefore, setting $x_{n}=z$ in (9), we have

$$
\begin{aligned}
d\left(x_{n+1},(T u)_{(\alpha, \beta)}\right) \leq & H\left(\left(T x_{n}\right)_{(\alpha, \beta)},(T u)_{(\alpha, \beta)}\right) \\
\leq & \alpha \max \left\{d\left(x_{n}, u\right), d\left(x_{n},\left(T x_{n}\right)_{(\alpha, \beta)}\right), d\left(u,(T u)_{(\alpha, \beta)}\right),\right. \\
& \left.\frac{d\left(x_{n},(T u)_{(\alpha, \beta)}\right)+d\left(u,\left(T x_{n}\right)_{(\alpha, \beta)}\right)}{2}, \frac{d\left(x_{n},\left(T x_{n}\right)_{(\alpha, \beta)}\right) d\left(u,(T u)_{(\alpha, \beta)}\right)}{1+d\left(x_{n}, u\right)}\right\} \\
+ & k \min \left\{d\left(x_{n},\left(T x_{n}\right)_{(\alpha, \beta)}\right), d\left(u,\left(T x_{n}\right)_{(\alpha, \beta)}\right), \frac{d\left(x_{n},\left(T x_{n}\right)_{(\alpha, \beta)}\right) d\left(u,(T u)_{(\alpha, \beta)}\right)}{1+d\left(x_{n}, u\right)}\right\} \\
\leq & \alpha \max \left\{d\left(x_{n}, u\right), d\left(x_{n}, x_{n+1}\right), d\left(u,(T u)_{(\alpha, \beta)}\right),\right. \\
& \left.\frac{d\left(x_{n},(T u)_{(\alpha, \beta)}\right)+d\left(u, x_{n+1}\right)}{2}, \frac{d\left(x_{n}, x_{n+1}\right) d\left(u,(T u)_{(\alpha, \beta)}\right)}{1+d\left(x_{n}, u\right)}\right\} \\
+ & \beta \min \left\{d\left(x_{n}, x_{n+1}\right), d\left(u, x_{n+1}\right), \frac{d\left(x_{n}, x_{n+1}\right) d\left(u,(T u)_{(\alpha, \beta)}\right)}{1+d\left(x_{n}, u\right)}\right\} .
\end{aligned}
$$

As $n \rightarrow \infty$ in the above inequalities, we have

$$
d\left(u,(T u)_{(\alpha, \beta)}\right) \leq \alpha \max \left\{d\left(u,(T u)_{(\alpha, \beta)}\right), \frac{d\left(u,(T u)_{(\alpha, \beta)}\right)}{2}, 0\right\} \leq \alpha d\left(u,(T u)_{(\alpha, \beta)}\right) .
$$

Since $1-\alpha>0$, therefore, (11) implies $d\left(u,(T u)_{(\alpha, \beta)}\right)=0$, and consequently, $u \in(T u)_{(\alpha, \beta)}$. 
Example 3. Let $X=\{4,5,6\},\{4\},\{5\},\{6\}$ be crisp sets. Define $d: X \times X \rightarrow \mathbb{R}$ as follows:

$$
d(x, y)= \begin{cases}0, & \text { if } x=y, \\ 7 / 20, & \text { if } x \neq y \text { and } x, y \in X \backslash\{5\} \\ 1, & \text { if if } x \neq y \text { and } x, y \in X \backslash\{6\} \\ 9 / 20, & \text { if } x \neq y \text { and } x, y \in X \backslash\{4\}\end{cases}
$$

Define a hesitant fuzzy mapping $T: X \rightarrow F_{H}(X)$ as follows:

$$
T(4)(t)=T(6)(t)=\left\{\begin{array}{ll}
\{0.6,0.8,1\}, & \text { if } t=4, \\
\{0.2,0.5,0.7\}, & \text { if } t=5, \\
\{0,0.3,0.4\}, & \text { if } t=6,
\end{array} \quad T(5)(t)= \begin{cases}\{0.1,0.4,0.5\}, & \text { if } t=4, \\
\{0.3,0.6,8\}, & \text { if } t=5, \\
\{0.5,0.7,0.9\}, & \text { if } t=6 .\end{cases}\right.
$$

For $x \in X \backslash\{5\}$, the score functions of $T$ corresponding to $t=4,5$ and $t=6$, are respectively given by $s((T x)(t))=(0.6+0.8+1) / 3=0.8, s((T x)(t))=(0.2+0.5+0.7) / 3=0.47$, $s((T x)(t))=(0+0.3+0.4) / 3=0.23$; and for $x \in X \backslash\{4,6\}$, the score functions of $T$ corresponding to $t=4,5$ and $t=6$, are respectively given by $s((T x)(t))=(0.1+0.4+0.5) / 3=$ $0.33, s((T x)(t))=(0.3+0.6+0.8) / 3=0.57, s((T x)(t))=(0.5+0.7+0.9) / 3=0.7$.

Then, for $\alpha=0.7$ and $\beta=0.16$, we have

$$
(T x)_{(\alpha, \beta)}=\{t \in X: s((T x)(t)) \geq \alpha\} \bigcup\{t \in X: s((T x)(t)) \leq \beta\}= \begin{cases}\{4\}, & \text { if } x \neq 5, \\ \{6\}, & \text { if } x=5 .\end{cases}
$$

Now, consider the following cases.

Case I. For $x \in X \backslash\{5\}, d\left(x,(T x)_{(\alpha, \beta)}\right)=\inf \left\{d(x, y): y \in(T x)_{(\alpha, \beta)}\right\}=0$. Hence, for $x=y$, we have $r(\alpha) d\left(x,(T x)_{(\alpha, \beta)}\right) \leq d(x, y)$.

Case II. For $x \in X \backslash\{4,6\}, d\left(x,(T x)_{(\alpha, \beta)}\right)=\inf \left\{d(x, y): y \in(T x)_{(\alpha, \beta)}\right\}=9 / 20$. Therefore, for $x \neq y$, we get $r(\alpha) d\left(x,(T x)_{(\alpha, \beta)}\right)=9 / 100 \leq d(x, y)$. Thus, from the two cases, it follows that there exists $(\alpha, \beta)=(0.7,0.16) \in(0,1] \times[0,1)$ such that $r(\alpha) d\left(x,(T x)_{(\alpha, \beta)}\right) \leq d(x, y)$ implies $H\left((T x)_{(\alpha, \beta)},(T y)_{(\alpha, \beta)}\right) \leq \alpha \vee(x, y)+\beta \wedge(x, y)$. Consequently, all the hypotheses of Theorem 3 are satisfied to obtain $4 \in(T 4)_{(0.7,0.16)}$.

Remark 1. It is clear that Theorem 3 cannot be followed from Theorem 2 due to T. Suzuki [23] even when $\beta=0$.

Corollary 1. Let $(X, d)$ be a complete metric space and $T: X \rightarrow F_{H}(X)$ be a hesitant fuzzy mapping. Assume that for $x, y \in X$ with $x \neq y$, there exists $(\alpha, \beta) \in(0,1] \times[0,1)$ such that $(T x)_{(\alpha, \beta)}$ is a nonempty closed and bounded subset of X. If $r(\alpha) d\left(x,(T x)_{(\alpha, \beta)}\right) \leq d(x, y)$ implies $H\left((T x)_{(\alpha, \beta)},(T y)_{(\alpha, \beta)}\right) \leq \alpha \vee(x, y)$, then $\mathcal{F}_{i x}(T) \neq \varnothing$.

Next, we present a local hesitant fuzzy fixed point theorem for Suzuki-type $(\alpha, \beta)$-weak contractions. First, recall that an open ball with radius $r>0$, centered at $x_{0}$ in a metric space $X$, is given by $B_{r}\left(x_{0}\right)=\left\{x \in X: d\left(x, x_{0}\right)<r\right\}$.

Theorem 4. Let $(X, d)$ be a complete metric space, $T: B_{r}\left(x_{0}\right) \rightarrow F_{H}(X)$ be a Suzuki-type $(\alpha, \beta)$-weak contraction. Assume that for each $x \in X$, there exists $(\alpha, \beta) \in(0,1] \times[0,1)$ such that $(T x)_{(\alpha, \beta)}$ is a nonempty closed and bounded subset of $X$, and $d\left(x_{0},\left(T x_{0}\right)_{(\alpha, \beta)}\right)<(1-\alpha) r$. Then $\mathcal{F}_{i x}(T) \neq \varnothing$. 
Proof. Let $0<\eta<r$ be such that $0<(1-\alpha)(1+\sqrt{\alpha}) \leq 1 /(1+\eta), B_{\eta}^{*}\left(x_{0}\right) \subset B_{r}\left(x_{0}\right)$ and $d\left(x_{0},\left(T x_{0}\right)_{(\alpha, \beta)}\right)<(1-\alpha) \eta$. Therefore, $(1-\alpha) \eta-d\left(x_{0},\left(T x_{0}\right)_{(\alpha, \beta)}\right)>0$. Choose

$$
\gamma=(1-\alpha) \eta-d\left(x_{0},\left(T x_{0}\right)_{(\alpha, \beta)}\right)>0
$$

then there exists $x_{1} \in\left(T x_{0}\right)_{(\alpha, \beta)}$ such that $d\left(x_{0}, x_{1}\right)<d\left(x_{0},\left(T x_{0}\right)_{(\alpha, \beta)}\right)+\gamma$. Thus, $d\left(x_{0}, x_{1}\right)<$ $(1-\alpha) \eta$. Now, for $\rho \sqrt{\alpha}=1$ and $x_{1} \in\left(T x_{0}\right)_{(\alpha, \beta)}$, there exists $x_{2} \in\left(T x_{1}\right)_{(\alpha, \beta)}$ such that $d\left(x_{1}, x_{2}\right) \leq \rho H\left(\left(T x_{0}\right)_{(\alpha, \beta)},\left(T x_{1}\right)_{(\alpha, \beta)}\right)$. Noting that

$$
r(\alpha) d\left(x_{0},\left(T x_{0}\right)_{(\alpha, \beta)}\right) \leq r(\alpha) d\left(x_{0}, x_{1}\right) \leq d\left(x_{0}, x_{1}\right),
$$

we have

$$
\begin{aligned}
d\left(x_{1}, x_{2}\right) \leq & \rho H\left(\left(T x_{0}\right)_{(\alpha, \beta)},\left(T x_{1}\right)_{(\alpha, \beta)}\right)=\frac{1}{\sqrt{\alpha}} H\left(\left(T x_{0}\right)_{(\alpha, \beta)},\left(T x_{1}\right)_{(\alpha, \beta)}\right) \\
\leq & \sqrt{\alpha} \vee\left(x_{0}, x_{1}\right)+\frac{\beta}{\sqrt{\alpha}} \wedge\left(x_{0}, x_{1}\right) \leq \sqrt{\alpha} \max \left\{d\left(x_{0}, x_{1}\right), d\left(x_{0},\left(T x_{0}\right)_{(\alpha, \beta)}\right), d\left(x_{1},\left(T x_{1}\right)_{(\alpha, \beta)}\right),\right. \\
& \left.\frac{d\left(x_{0},\left(T x_{1}\right)_{(\alpha, \beta)}\right)+d\left(x_{1},\left(T x_{0}\right)_{(\alpha, \beta)}\right)}{2}, \frac{d\left(x_{0},\left(T x_{0}\right)_{(\alpha, \beta)}\right) d\left(x_{1},\left(T x_{1}\right)_{(\alpha, \beta)}\right)}{1+d\left(x_{0}, x_{1}\right)}\right\} \\
+ & \frac{\beta}{\sqrt{\alpha}} \min \left\{d\left(x_{0},\left(T x_{0}\right)_{(\alpha, \beta)}\right), d\left(x_{1},\left(T x_{0}\right)_{(\alpha, \beta)}\right), \frac{d\left(x_{0},\left(T x_{0}\right)_{(\alpha, \beta)}\right) d\left(x_{1},\left(T x_{1}\right)(\alpha, \beta)\right.}{1+d\left(x_{0}, x_{1}\right)}\right\} \\
\leq & \sqrt{\alpha} \max \left\{d\left(x_{0}, x_{1}\right), d\left(x_{0}, x_{1}\right), d\left(x_{1}, x_{2}\right), \frac{d\left(x_{0}, x_{2}\right)+d\left(x_{1}, x_{1}\right)}{2}, \frac{d\left(x_{0}, x_{1}\right) d\left(x_{1}, x_{2}\right)}{1+d\left(x_{0}, x_{1}\right)}\right\} \\
& +\frac{\beta}{\sqrt{\alpha}} \min \left\{d\left(x_{0}, x_{1}\right), d\left(x_{1}, x_{1}\right), \frac{d\left(x_{0}, x_{1}\right) d\left(x_{1}, x_{2}\right)}{1+d\left(x_{0}, x_{2}\right)}\right\} \\
\leq & \sqrt{\alpha} \max \left\{d\left(x_{0}, x_{1}\right), d\left(x_{1}, x_{2}\right), \frac{d\left(x_{0}, x_{1}\right)+d\left(x_{1}, x_{2}\right)}{2}\right\}+\frac{\beta}{\sqrt{\alpha}} \min \left\{d\left(x_{0}, x_{1}\right), 0, d\left(x_{1}, x_{2}\right)\right\} \\
\leq & \sqrt{\alpha} \max \left\{d\left(x_{0}, x_{1}\right), d\left(x_{1}, x_{2}\right)\right\} .
\end{aligned}
$$

If $d\left(x_{0}, x_{1}\right) \leq d\left(x_{1}, x_{2}\right)$, then the above gives $d\left(x_{1}, x_{2}\right) \leq \sqrt{\alpha} d\left(x_{1}, x_{2}\right)<d\left(x_{1}, x_{2}\right)$, a contradiction. Hence, $d\left(x_{0}, x_{1}\right)>d\left(x_{1}, x_{2}\right)$ and $d\left(x_{1}, x_{2}\right)<\sqrt{\alpha} d\left(x_{0}, x_{1}\right) \sqrt{\alpha}(1-\alpha) \eta$. Note that by assumption and the triangle inequality, we have

$$
d\left(x_{0}, x_{2}\right) \leq d\left(x_{0}, x_{1}\right)+d\left(x_{1}, x_{2}\right)<(1-\alpha) \eta+\sqrt{\alpha}(1-\alpha) \eta \leq \frac{\eta}{1+\eta}<\eta .
$$

Hence, $x_{2} \in B_{\eta}\left(x_{0}\right)$. Continuing this process recursively, we generate a sequence $\left\{x_{n}\right\}_{n \in \mathbb{N}}$ such that

(a) $x_{n} \in B_{\eta}\left(x_{0}\right)$ for all $n \in \mathbb{N}$;

(b) $x_{n} \in\left(T x_{n}\right)_{(\alpha, \beta)}$ for each $n \in \mathbb{N}$;

(c) $d\left(x_{n}, x_{n+1}\right) \leq(\sqrt{\alpha})^{n}(1-\alpha) \eta$ for all $n \in \mathbb{N}$.

From (c), it follows by usual arguments that $\left\{x_{n}\right\}_{n \in \mathbb{N}}$ is a Cauchy sequence and converges to some $u \in B_{r}\left(x_{0}\right)$. From here, following the steps in the proof of Theorem 3, we conclude that $\mathcal{F}_{i x}(T) \neq \varnothing$. 


\section{Further consequences}

In this section, we show that there is a link between crisp multi-valued and single-valued mappings and hesitant fuzzy set-valued mappings by establishing some consequences of Theorem 3.

Corollary 2. Let $(X, d)$ be a complete metric space and $\Lambda: X \rightarrow C B(X)$ be a multi-valued mapping. Assume that for all $x, y \in X$, there exists $(\alpha, \beta) \in(0,1] \times[0,1)$ such that $r(\alpha) d(x, \Lambda x) \leq$ $d(x, y)$ implies $H(\Lambda x, \Lambda y) \leq \alpha \vee(x, y)+\beta \wedge(x, y)$, where the mapping $r: I_{-1} \rightarrow I^{+1}$ is defined by

$$
r(\alpha)= \begin{cases}1, & \text { if } \quad 0 \leq \alpha<1 / 2 \\ 1-\alpha, & \text { if } \quad 1 / 2 \leq \alpha<1\end{cases}
$$

Then $\mathcal{F}_{i x}(\Lambda) \neq \varnothing$.

Proof. Let $I^{+}=(0,1]$ and $P\left(I^{+}\right)$be the power set of $I^{+}$. Consider a mapping $\omega: X \rightarrow P\left(I^{+}\right)$ and a hesitant fuzzy mapping $T: X \rightarrow F_{H}(X)$ defined by

$$
(T x)(t)= \begin{cases}\omega(x) \backslash\{1\}, & \text { if } t \in \Lambda x, \\ \{1\}, & \text { if } t \notin \Lambda x .\end{cases}
$$

Let $\alpha=\inf \{s((T x)(t)): t \in X\}$ and $\beta=0$. Then,

$$
(T x)_{(\alpha, \beta)}=\{t \in X: s((T x)(t)) \geq \alpha\} \bigcup\{t \in X: s((T x)(t)) \leq \beta\}=\Lambda x .
$$

Therefore, $d(x, y) \geq r(\alpha) d(x, \Lambda x)=r(\alpha) d\left(x,(T x)_{(\alpha, \beta)}\right)$. Thus, Theorem 3 can be applied to find $u \in X$ such that $u \in \Lambda u$.

In the following, we provide an example to highlight the generality of Corollary 2.

Example 4. Let $X=\{a, b, c, p, q\}$ and $d: X \times X \rightarrow[0, \infty)$ be defined by $d(a, b)=d(a, c)=7$, $d(b, q)=d(c, p)=d(c, q)=d(b, c)=12, d(a, p)=d(a, q)=16, d(b, p)=14, d(p, q)=8$, $d(u, u)=0$ and $d(u, v)=d(v, u)$ for all $u, v \in X$. Define $\Lambda: X \rightarrow C B(X)$ by

$$
\Lambda u=\left\{\begin{array}{lll}
\{c\}, & \text { if } & u \in X \backslash\{a, b, c, p\}, \\
\{a, b\}, & \text { if } \quad u \in X \backslash\{a, b, c, q\}, \\
\{a\}, & \text { if } u \in X \backslash\{p, q\} .
\end{array}\right.
$$

Now, it is easy to verify that all the hypotheses of Corollary 2 are satisfied with $\alpha=13 / 16$ and $\beta=0.5$. In fact, $r(\alpha) d(p, \Lambda p)=5.25 \leq d(p, q)$ implies

$$
\begin{aligned}
H(\Lambda p, \Lambda q)=H(\{a, b\},\{c\})=12 & \leq \alpha \vee(p, q)+\beta \wedge(p, q) \\
& \leq \frac{13}{16} \max \{8,12,12,12,16\}+0.5 \min \{12,12,16\} \leq 19 .
\end{aligned}
$$

Further, we see that there exists $u=a \in X$ such that $u \in \Lambda u$. On the other hand, observe that if we set $u=p$ and $v=q$, then

$$
\begin{aligned}
\vee(p, q) & =\max \left\{d(p, q), d(p, \Lambda p), d(q, \Lambda q), \frac{d(p, \Lambda q)+d(q, \Lambda p)}{2}, \frac{d(p, \Lambda p) d(q, \Lambda q)}{1+d(p, q)}\right\} \\
& =\max \{8,12,12,12,16\}=16,
\end{aligned}
$$

and $H(\Lambda p, \Lambda q)=12>10=\alpha \vee(p, q)$. Therefore, [12, Theorem 2.1] and [10, Theorem 3] are not applicable in this case to find $u \in \Lambda u$. 
Corollary 3. Let $(X, d)$ be a complete metric space and $\Lambda: X \rightarrow X$ be a single-valued mapping. Assume that for all $x, y \in X$, there exists $(\alpha, \beta) \in(0,1] \times[0,1)$ such that $r(\alpha) d(x, \Lambda x) \leq d(x, y)$ implies $d(\Lambda x, \Lambda y) \leq \alpha \vee(x, y)+\beta \wedge(x, y)$, where the mapping $r: I_{-1} \rightarrow I^{+1}$ is defined by

$$
r(\alpha)= \begin{cases}1, & \text { if } \quad 0 \leq \alpha<1 / 2 \\ 1-\alpha, & \text { if } \quad 1 / 2 \leq \alpha<1\end{cases}
$$

Then, there exists $u \in X$ such that $\Lambda u=u$.

Proof. We know that $\{x\} \in C B(X)$ for every $x \in X$. Consider a mapping $\Xi: X \rightarrow C B(X)$ defined as $\Xi x=\{\Lambda x\}, x \in X$. Then all the conditions of Corollary 2 reduce to the conditions of Corollary 3 with $H(\Lambda x, \Lambda y)=d(\Lambda x, \Lambda y)$ for all $x, y \in X$. Thus, by application of Corollary 2, there exists $u \in X$ such that $\{u\}=\Xi u$. The definition of $\Xi$ implies that $\Xi u=\{\Lambda u\}$. Consequently, $u=\Lambda u$.

By using the method of proving Corollary 2 and applying Theorem 3, we can also derive the following results.

Corollary 4 ([12, Theorem 2.1]). Let $(X, d)$ be a complete metric space and $\Lambda: X \rightarrow C B(X)$ be a multi-valued mapping. Assume that there exists an $\alpha \in(0,1]$ such that for all $x, y \in X$, $r(\alpha) d(x, \Lambda x) \leq d(x, y)$ implies $H(\Lambda x, \Lambda y) \leq \alpha d(x, y)$, where the mapping $r:(0,1) \rightarrow(0,1]$ is defined by

$$
r(\alpha)= \begin{cases}1, & \text { if } \quad 0 \leq \alpha<1 / 2 \\ 1-\alpha, & \text { if } \quad 1 / 2 \leq \alpha<1\end{cases}
$$

Then there exists $u \in X$ such that $u \in \Lambda u$.

Corollary 5 ([12]). Let $(X, d)$ be a complete metric space and $\Lambda: X \rightarrow C B(X)$ be a multivalued mapping. Assume that there exists $\alpha \in(0,1)$ such that $r(\alpha) d(x, \Lambda x) \leq d(x, y)$ implies $H(\Lambda x, \Lambda y) \leq \alpha \max \{d(x, y), d(x, \Lambda x), d(y, \Lambda y)\}$ for all $x, y \in X$, where the function $r$ is defined as in Corollary 2. Then, there exists $u \in X$ such that $u \in \Lambda u$.

Corollary 6 ([9]). Let $(X, d)$ be a complete metric space and $\Lambda: X \rightarrow C B(X)$ be a multi-valued mapping. Let the function $r: I_{-1} \rightarrow I^{+1}$ be as defined in Corollary 2. Assume that there exists $a(x, y) \in[0,1)$ such that $r(\alpha) d(x, \Lambda x) \leq d(x, y)$ implies $H(\Lambda x, \Lambda y) \leq a(x, y)[d(x, \Lambda y)+$ $d(y, \Lambda x)]$ for all $x, y \in X$. Then there exists $u \in X$ such that $u \in \Lambda u$.

\section{Application in dynamic programming}

There are more than a handful of literature dealing with the study of functional equations arising in dynamic programming. The interested reader may consult [4] and the references therein for detail analysis on this topic. Hereafter, let $M$ and $Q$ be Banach spaces and $F \subseteq M$, $G \subseteq Q$, where $F$ and $G$ are the state and decision spaces, respectively. Recall that a state space is the set of all feasible state and a decision space is the resultant network formed by the nodes of feasible states and all the feasible decisions. Let $B(F)$ be the set of all bounded real-valued functions defined on $F$. It is well-known that $B(F)$ equipped with the metric $d$, given as $d(p, q)=\sup _{x \in F}|p(x)-q(x)|$, for all $p, q \in B(F)$ is a complete metric space. The classical form of functional equation arising in dynamic programming is given by (see [4]) 
$h(x)=o_{p t} H(x, y, h(\kappa(x, y)))$, where $x \in F$ and $y \in G$ are the state and decision vectors, respectively, the opt denotes either sup, inf, max or $\min , \kappa: F \times G \rightarrow F$ is the transformation of the process and $h(x)$ represents the optimal return function with initial state $x$.

Our aim in this section is to apply Corollary 3 to find the optimal decision in the given state space using dynamic programming in connection with the problem of solving the functional equation, given as

$$
h(x)=\sup \{\pi(x, y)+\Theta(x, y, h(\kappa(x, y))): x \in F, y \in G\},
$$

where $\pi: F \times G \rightarrow \mathbb{R}$ and $\Theta: F \times G \times \mathbb{R} \rightarrow \mathbb{R}$ are bounded functions. Let the function $r$ be as given in Corollary 3. Define the mapping $T: B(F) \rightarrow B(F)$ by

$$
T(p(x))=\sup \{\pi(x, y)+\Theta(x, y, h(\kappa(x, y))): x \in F, y \in G\} .
$$

Theorem 5. Assume that there exists $(\alpha, \beta) \in(0,1] \times[0,1)$ such that for all $(x, y) \in F \times G$, $p, q \in B(F)$ and $\lambda \in F$, the inequality $r(\alpha) d(p, T(p)) \leq d(p, q)$ implies

$$
|\Theta(x, y, p(\lambda))-\Theta(x, y, q(\lambda))| \leq \alpha \vee(p(\lambda), q(\lambda))+\beta \wedge(p(\lambda), q(\lambda)),
$$

where

$$
\vee(p(\lambda), q(\lambda))=\max \left\{d(p, q), d(p, T p), d(q, T q), \frac{d(p, T q)+d(q, T p)}{2}, \frac{d(p, T p) d(q, T q)}{1+d(p, q)}\right\},
$$

and

$$
\wedge(p(\lambda), q(\lambda))=\min \left\{d(p, T p), d(q, T p), \frac{d(p, T p) d(q, T q)}{1+d(p, q)}\right\} .
$$

Then, the functional equation (12) has a unique bounded solution in $B(F)$.

Proof. Note that $T$ is a self-map on $B(F)$. Let $\tau$ be an arbitrary positive real and $p_{1}, p_{2} \in B(F)$. For $x \in F$, let $y_{1}, y_{2} \in G$ be chosen such that

$$
\begin{aligned}
& T\left(p_{1}(x)\right)<\pi\left(x, y_{1}\right)+\Theta\left(x, y_{1}, p_{1}\left(\kappa_{1}\right)\right)+\tau, \\
& T\left(p_{2}(x)\right)<\pi\left(x, y_{2}\right)+\Theta\left(x, y_{2}, p_{2}\left(\kappa_{2}\right)\right)+\tau,
\end{aligned}
$$

where $\kappa_{1}=\kappa\left(x, y_{1}\right)$ and $\kappa_{2}=\kappa\left(x, y_{2}\right)$. From (13), we get

$$
\begin{aligned}
& T\left(p_{1}(x)\right) \geq \pi\left(x, y_{2}\right)+\Theta\left(x, y_{2}, p_{1}\left(\kappa_{2}\right)\right), \\
& T\left(p_{2}(x)\right) \geq \pi\left(x, y_{1}\right)+\Theta\left(x, y_{1}, p_{2}\left(\kappa_{1}\right)\right) .
\end{aligned}
$$

From (14) and (17), we have

$$
\begin{aligned}
T\left(p_{1}(x)\right)-T\left(p_{2}(x)\right) & <\Theta\left(x, y_{1}, p_{1}\left(\kappa_{1}\right)\right)-\Theta\left(x, y_{1}, p_{2}\left(\kappa_{1}\right)\right)+\tau \\
& \leq \mid \Theta\left(x, y_{1}, p_{1}\left(\kappa_{1}\right)\right)-\Theta\left(x, y_{1}, p_{2}\left(\kappa_{1}\right)\right)+\tau \\
& \leq \alpha \vee\left(p_{1}(x), p_{2}(x)\right)+\beta \wedge\left(p_{1}(x), p_{2}(x)\right)+\tau .
\end{aligned}
$$

On similar steps, from (15) and (16), we obtain

$$
T\left(p_{2}(x)\right)-T\left(p_{1}(x)\right) \leq \alpha \vee\left(p_{1}(x), p_{2}(x)\right)+\beta \wedge\left(p_{1}(x), p_{2}(x)\right)+\tau .
$$

Thus, from (18) and (19), we have

$$
\left|T\left(p_{1}(x)\right)-T\left(p_{2}(x)\right)\right| \leq \alpha \vee\left(p_{1}(x), p_{2}(x)\right)+\beta \wedge\left(p_{1}(x), p_{2}(x)\right)+\tau .
$$

Since $\tau$ is arbitrary and (20) holds for all $x \in F$, hence $r(\alpha) d\left(p_{1}, T\left(p_{1}\right)\right) \leq d\left(p_{1}, p_{2}\right)$ implies $d\left(T\left(p_{1}\right), T\left(p_{2}\right)\right) \leq \alpha \vee\left(p_{1}, p_{2}\right)+\beta \wedge\left(p_{1}, p_{2}\right)$. We see that all the conditions of Corollary 3 are satisfied. It follows that the functional equation (12) has a unique bounded solution. 


\section{Conclusion}

In this note, the idea of Suzuki-type $(\alpha, \beta)$-weak contractions in the setting of hesitant fuzzy set-valued maps is proposed. Thereafter, we established hesitant fuzzy fixed point theorem which is a generalization of some comparable results in the literature. As corollaries, a few well-known results of crisp multivalued and single-valued mappings are obtained. Moreover, an application in dynamic programming is considered to indicate a possible usability of our obtained results. The presented notions in this work can be further extended to study a more generalized concepts of hesitant fuzzy sets and other hybrid models in the literature of both fuzzy and classical mathematics.

\section{Acknowledgement}

The authors are thankful to the editors and the anonymous reviewers for their valuable suggestions and comments that helped to improve this manuscript.

\section{References}

[1] Abu-Donia H.M. Common fixed point theorems for fuzzy mappings in metric space under $\varphi$-contraction condition. Chaos Solitons Fractals 2007, 34 (2), 538-543. doi:10.1016/j.chaos.2005.03.055

[2] Azam A., Fisher B., Khan M. Common fixed point theorems in complex valued metric spaces. Numer. Funct. Anal. Optim. 2011, 32 (3), 243-253.

[3] Banach S. Sur les opérations dans les ensembles abstraits et leur application aux équations intéxgrales. Fund. Math. 1922, 3 (1), 133-181.

[4] Bellman R., Lee E.S. Functional equations arising in dynamic programming. Aequationes Math. 1978, 17, 1-18.

[5] Berinde V. Approximating fixed points of weak contractions using the Picard iteration. Nonlinear Anal. Forum $2004,9,43-54$.

[6] Berinde M., Berinde V. On a general class of multi-valued weakly Picard mappings. J. Math. Anal. Appl. 2007,326 (2), 772-782. doi:10.1016/j.jmaa.2006.03.016

[7] Borisut P., Kumam P., Gupta V., Mani N. Generalized $(\psi, \alpha, \beta)$-weak contractions for initial value problems. Mathematics 2019, 7 (3), 266. doi:10.3390/math7030266

[8] Caristi J. Fixed point theorems for mappings satisfying inwardness conditions. Trans. Amer. Math. Soc. 1976, 215, 241-251. doi:10.2307/1999724

[9] Chandra N., Joshi M.C., Singh N.K. Fixed point theorems for generalized multivalued contraction. J. Anal. 2018, 26 (1), 49-59. doi:10.1007/s41478-017-0067-0

[10] Ćirić Lj.B. Fixed points for generalized multi-valued contractions. Mat. Vesnik 1972, 9 (24), 265-272.

[11] Ćirić Lj.B. A generalization of Banach's contraction principle. Proc. Amer. Math. Soc. 1974, 45 (2), $267-273$. doi:10.2307/2040075

[12] Đorić D., Lazović R. Some Suzuki-type fixed point theorems for generalized multivalued mappings and applications. Fixed Point Theory Appl. 2011, 2011 (1), 40. doi:10.1186/1687-1812-2011-40

[13] Frigon M., O'Regan D. Fuzzy contractive maps and fuzzy fixed points. Fuzzy Sets and Systems 2002,129 (1), 39-45. doi:10.1016/S0165-0114(01)00171-3

[14] Gupta V., Kumar S.R., Kanwar A. Some coupled fixed point results on modified intuitionistic fuzzy metric spaces and application to integral type contraction. Iran. J. Fuzzy Syst. 2017, 14 (5), 123-137. doi:10.22111/ijfs.2017.3436

[15] Heilpern S. Fuzzy mappings and fixed point theorem. J. Math. Anal. Appl. 1981, 83 (2), 566-569. doi:10.1016/0022247X(81)90141-4 
[16] Kannan R. Some results on fixed points - II. Amer. Math. Monthly 1969, 76 (4), 405-408. doi:10.2307/2316437

[17] Mohammed S.S. On Bilateral fuzzy contractions. Funct. Anal. Approx. Comput. 2020, 12 (1), 1-13.

[18] Mohammed S.S., Azam A. Fixed points of soft-set valued and fuzzy set-valued maps with applications. J. Intell. Fuzzy Syst. 2019, 37 (3), 3865-3877.

[19] Nadler S.B. Multi-valued contraction mappings. Pac. J. Math. 1969, 30 (2), 475-488.

[20] Rashid T., Beg I. Convex hesitant fuzzy sets. J. Intell. Fuzzy Syst. 2016, 30 (5), 2791-2796.

[21] Rodriguez R.M., Martinez L., Torra V., Xu Z.S., Herrera F. Hesitant fuzzy sets: state of the art and future directions. Int. J. Intell. Syst. 2014, 29 (6), 495-524. doi:10.1002/int.21654

[22] Subrahmanyam P.V. Remarks on some fixed point theorems related to Banach's contraction principle. J. Math. Phys. Sci. 1974, 8, 445-457.

[23] Suzuki T. A generalized Banach contraction principle that characterizes metric completeness. Proc. Amer. Math. Soc. 2008, 136 (5), 1861-1869. doi:10.1090/S0002-9939-07-09055-7

[24] Torra V. Hesitant fuzzy sets. Int. J. Intell. Syst. 2010, 25 (6), 529-539. doi:10.1002/int.20418

[25] Xia M., Xu Z. Hesitant fuzzy information aggregation in decision making. Internat. J. Approx. Reason. 2011, 52 (3), 395-407. doi:10.1016/j.ijar.2010.09.002

[26] Zadeh L.A. Fuzzy sets. Inf. Control 1965, 8 (3), 338-353. doi:10.1016/S0019-9958(65)90241-X

[27] Zhou L., Wu W.Z., Zhang W.X. Properties of the cut-sets of intuitionistic fuzzy relations. Fuzzy Syst. Math. 2009, $23(2), 110-115$.

Received 11.11.2020

Revised 22.03.2021

Шагарі М.Ш., Азам А. Нерухомі точки коливних нечітких багатозначних відображень та їх застосування // Карпатські матем. публ. - 2021. - Т.13, №3. - С. 711-726.

У цій роботі вводиться поняття коливних нечітких нерухомих точок. 3 цією метою означено $(\alpha, \beta)$-слабкий стиск типу Сузукі у рамках коливних нечітких багатозначних відображень, що, тим самим, встановлює деякі відповідні теореми про нерухомі точки. Введене поняття $\epsilon$ розширенням нечітких багатозначних відображень у відповідній літературі. Наведено приклади, які додатково підтверджують результати та загальність запропонованих ідей. Більше того, один із результатів використовується для дослідження достатніх умов існування класу функціональних рівнянь, що виникають у динамічному програмуванні.

Ключові слова і фрази: коливна нечітка множина, коливне нечітке багатозначне відображення, нерухома точка, слабкий стиск. 